\section{Atom-Probe Microscopy LEAPs the Chasm to Mainstream Applications}

\author{
Roger Alvis ${ }^{\star}$ and Thomas F. Kelly ${ }^{\star *}$ \\ ${ }^{*}$ FEI Company and ${ }^{*}$ Imago Scientific Instruments \\ Roger.Alvis@fei.com
}

In the now classic marketing text, Crossing the Chasm, Geoffrey Moore describes the difficult transition every successful new technology must make as it evolves from laboratory curiosity to mainstream acceptance. Essentially the transition consists of a radical shift in focus, from exploring the possibilities that fill the minds of visionaries and innovators to satisfying the practical concerns of pragmatists and conservatives who are more interested in integration with existing technologies and return on investment. Initial development of the atom probe was motivated primarily by scientific curiosity and the desire to do novel research with a new microscopy technique. As the technology matured the focus of development efforts shifted to expanding the applications space and improving practical performance in areas such as sensitivity, fidelity, productivity, and ease of use. These efforts also satisfied important requirements for mainstream acceptance: validating accuracy through comparisons with existing technologies and identifying high-value applications for its unique capabilities. Atom-probe microscopy has crossed the chasm to mainstream research and industrial applications, transforming itself from a laboratory curiosity to an essential capability in worldclass microscopy centers.

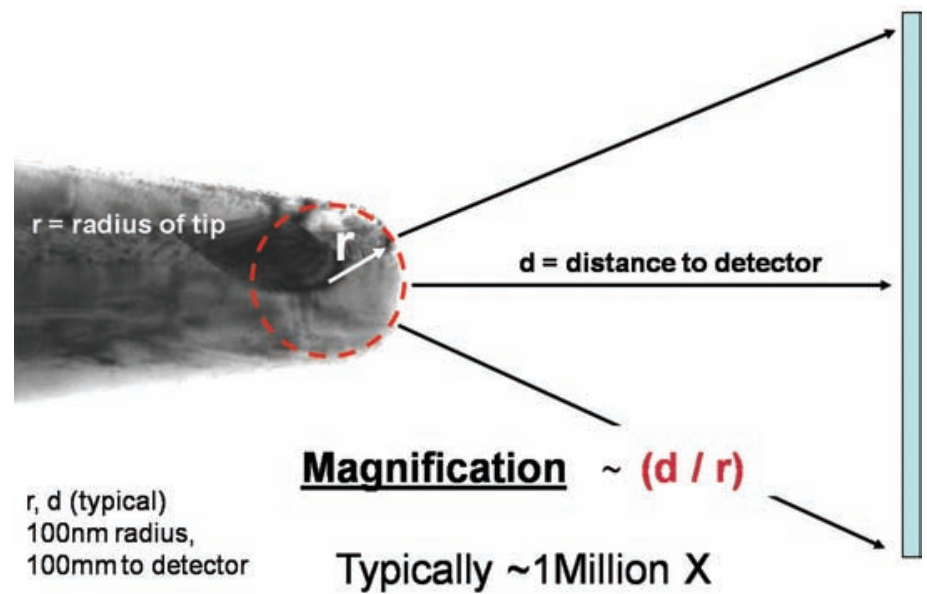

Figure 1 Point projection microscope

\section{Local-Electrode Atom-Probe (LEAP) Technology}

Atom-probe microscopy [1] integrates a point-projection microscope with a two-dimensional time-of-flight (TOF) mass spectrometer for combined imaging and compositional analysis. The sample is prepared as a finely pointed tip with a carefully shaped radius of approximately $100 \mathrm{~nm}$. Samples are frequently shaped with a focused ion beam (FIB), which provides fast, precise, reliable control of tip shape and is suitable for most materials. In the atomprobe microscope, ions field evaporated from the tip surface are accelerated by an electric field and follow diverging paths toward a position-sensitive detector where their landing positions map their original positions on the tip surface (figure 1). Magnifications of one million times are typical.

Evaporation can be triggered by a voltage or laser pulse. Voltage pulsing is limited to conductive materials. Laser pulsing extends the application space, in principle, to nearly any material. The pulse defines the beginning of the TOF interval in the mass spectrometer. The same electric field and detector that form the microscope also constitute the TOF spectrometer. The time required for caun ivin (or ionized molecule or molecular fragment) to reach the detector is determined by its mass-to-charge state ratio: lighter ions are accelerated by the electric field faster than heavier ions. TOF spectrometry permits detailed analysis of isotopic, elemental and chemical composition.
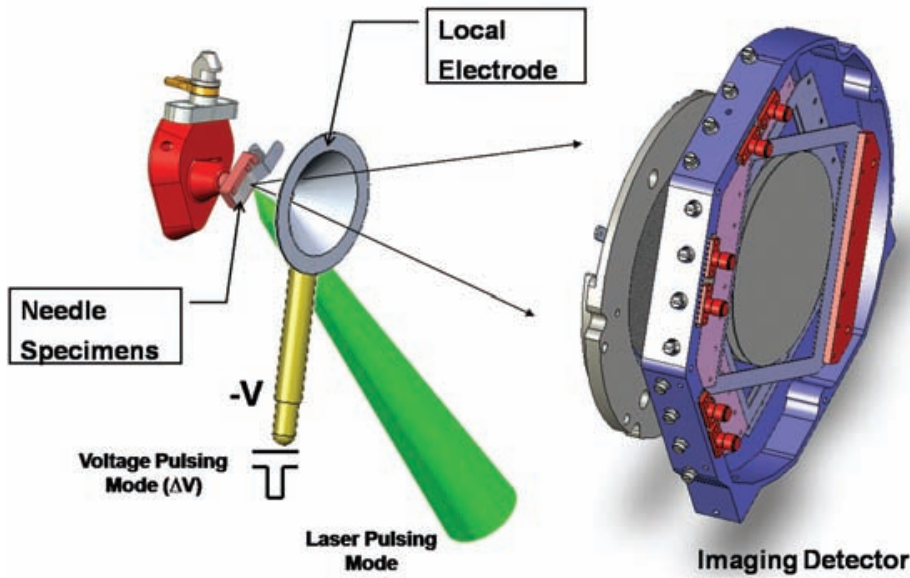

Figure 2 Local-electrode atom probe (LEAP)

In local-electrode atom probe (LEAP ${ }^{\circledR}$ ) configurations (figure 2) the accelerating field is formed by a cone-shaped electrode with a small aperture at its apex positioned very close (local) to the sample tip [2]. The local electrode reduces the voltage required to ionize atoms at the sample surface and provides a wider field of view than conventional atom-probe instruments. LEAP systems can be optimized for high mass resolution in voltage-pulse mode by altering and lengthening the flight path with an electrostatic mirror (a reflectron), or for improved imaging with a larger detector. Both configurations provide good mass resolution in laser pulse mode, and wide fields of view. In this article, unless otherwise indicated, the term atom-probe and all data and discussion refer to the LEAP configuration [3].

Field evaporation is a localized phenomenon and an evaporated ion's landing position $(\mathrm{X}, \mathrm{Y})$ on the detector corresponds to its starting location on tip surface. Evaporation events occur roughly once for every hundred pulses, allowing ions to pass through the TOF spectrometer one at a time. Since the evaporation occurs only from the tip surface and that surface erodes more or less uniformly, the pulse sequence provides a third spatial dimension $(Z)$. Thus the
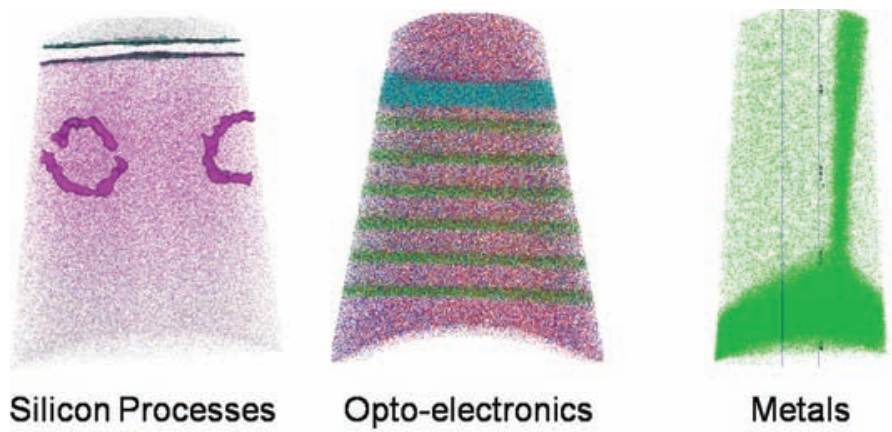

Metals
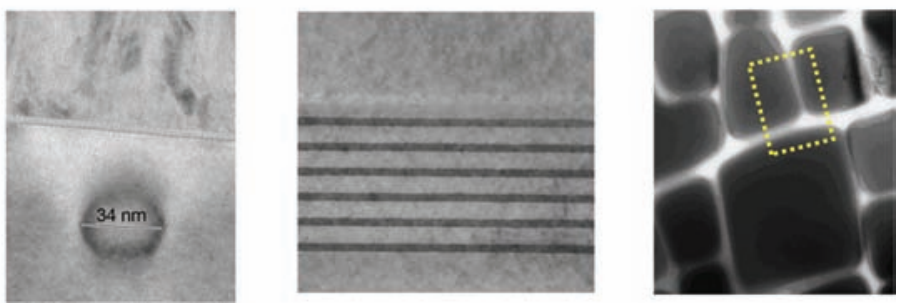

Figure 3 Atom maps and corresponding TEM images [4, 5] 


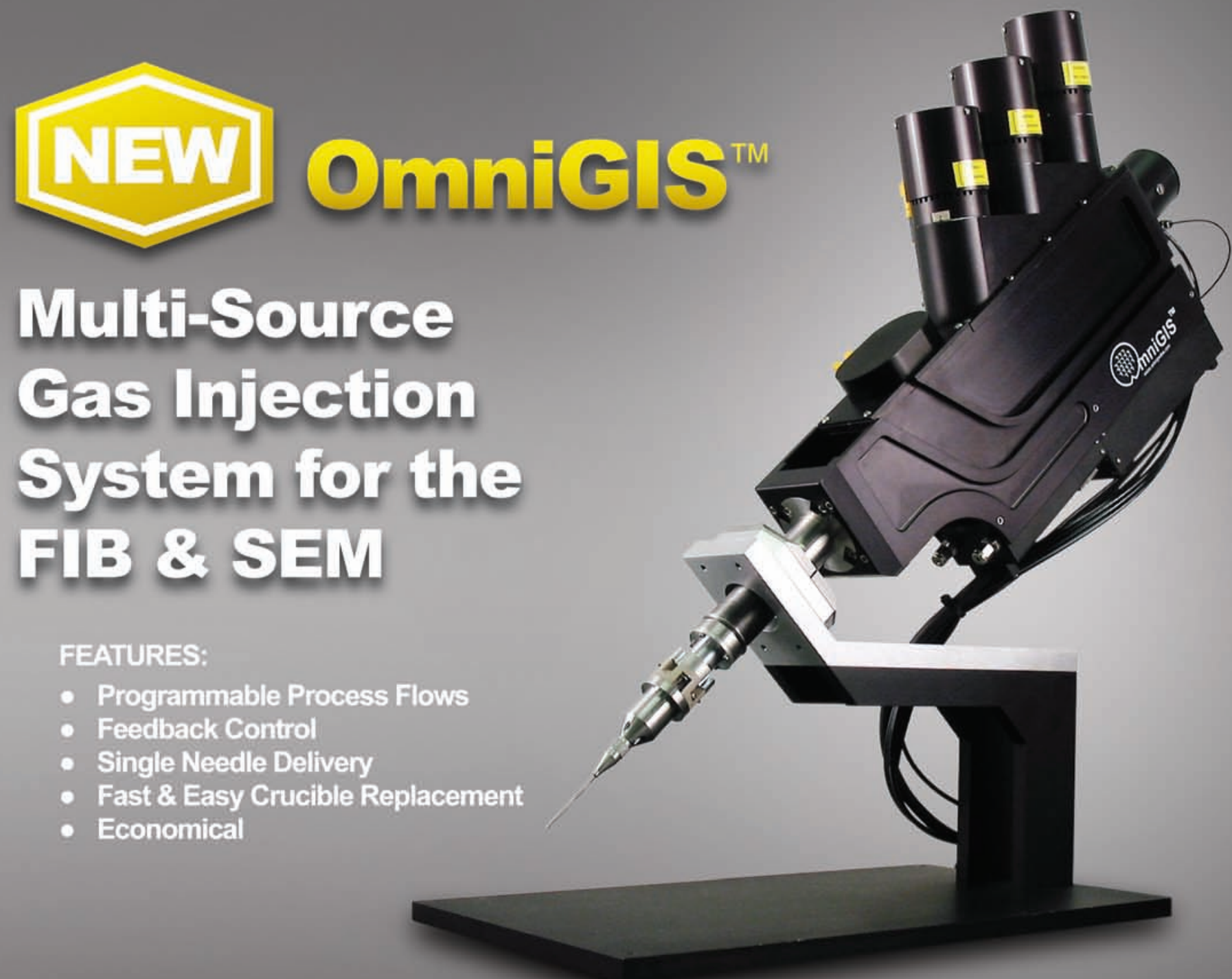

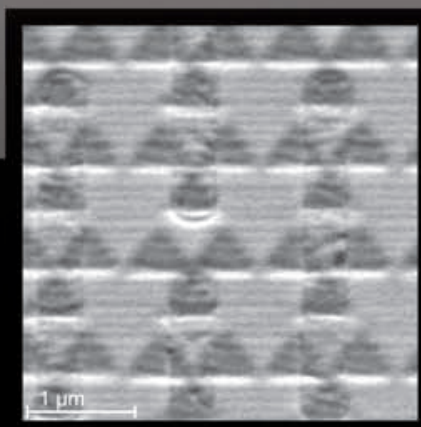

\section{Make}

FIB deposited plasmonic nanostructures
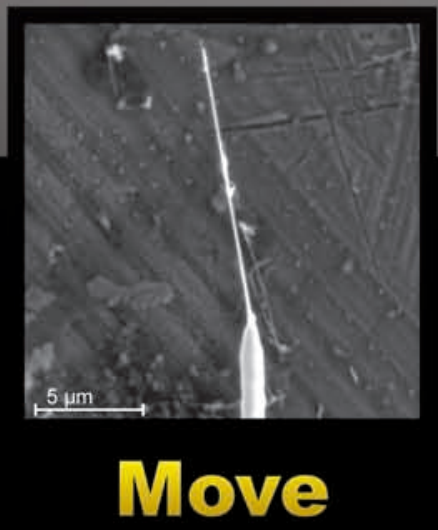

Single nanowire analysis

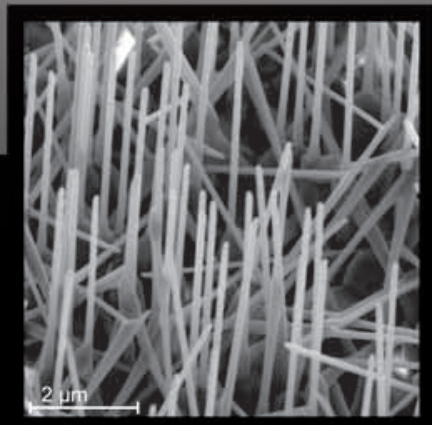

Modify

Chemical surface alteration

For more information visit us at http://www.omniprobe.com or call us at $\mathbf{2 1 4 . 5 7 0 . 6 8 0 0 .}$

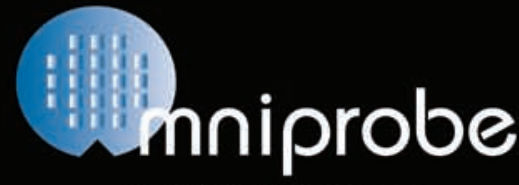




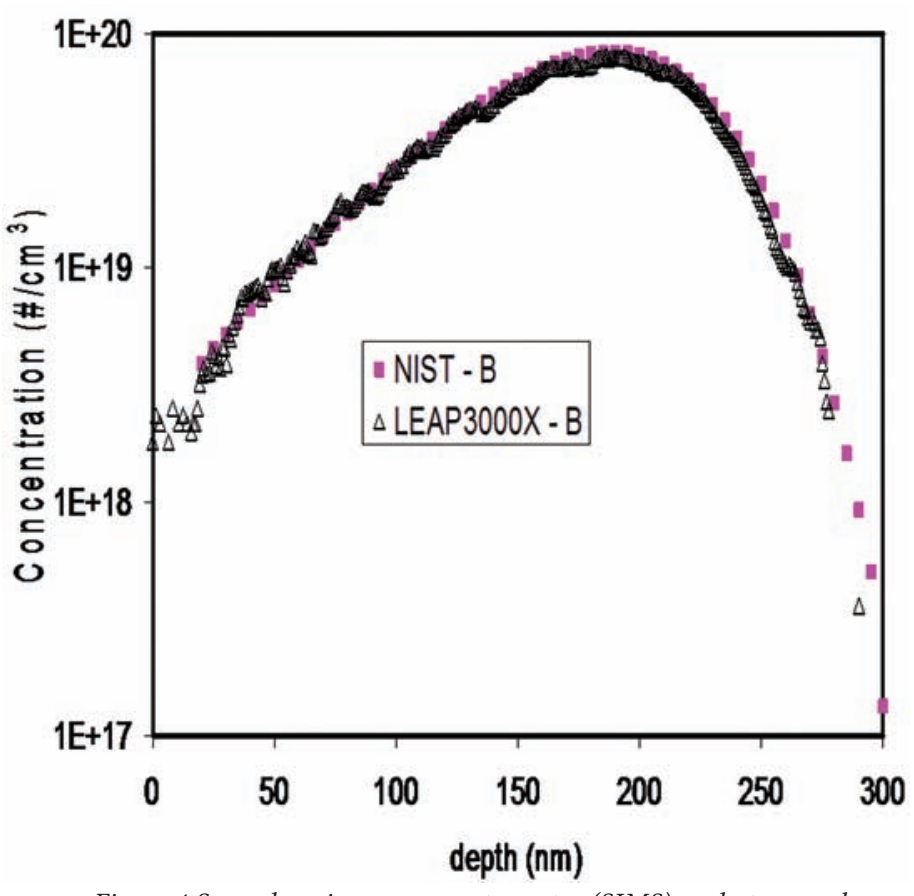

Figure 4 Secondary-ion mass spectrometry (SIMS) and atom-probe depth profiles for boron in silicon

entire data set consists of the $\mathrm{X}, \mathrm{Y}$ landing position on the detector, the pulse number and the time of flight interval for each evaporation event. The information it encodes constitutes a 3.1 dimensional, virtual microstructure, comprising the three spatial dimensions (X, $\mathrm{Y}$ and $\mathrm{Z}$ ) plus a compositional dimension (mass-to-charge-state ratio) for every detected ion. The information is presented visually as a 3-dimensional spatial model using color for the compositional dimension. It is easily analyzed along any combination of dimensions, for example: X, Y atom maps (2.1d), linear profiles in depth or any other direction (1.1d), and bulk composition (0.1d).

\section{Correlation Establishes Confidence}

Before any new analytical technique can gain acceptance it must demonstrate accuracy in comparison with known techniques on well characterized standards, documenting areas of agreement and, perhaps more importantly, identifying and understanding points of discrepancy. The accepted standard for high-resolution imaging is transmission electron microscopy (TEM), which has recently shown the ability to resolve atomic structure down to $0.5 \AA$. Figure 3 compares atom-probe maps and TEM images of several familiar structures in a variety of applications. They agree quite well for structures with dimensions ranging from $100 \mathrm{~nm}$ to $30 \mathrm{~nm}$.

Secondary-ion mass spectroscopy (SIMS) is widely accepted for measuring low-level concentrations of atomic species. It is used extensively to determine the depth distribution of impurities intention- ally introduced into the near surface region of the crystalline silicon substrate during semiconductor device fabrication. Precise control of these depth profiles is critical for proper electrical function. Figure 4 compares SIMS and atom-probe depth profiles of boron implanted into silicon acquired from a NIST standard (SRM 2137). Close agreement between the overlaid depth profiles confirms the accuracy of atom-probe analysis for concentrations ranging from $\sim 10^{18}$ up to $10^{20}$ atoms per $\mathrm{cm}^{3}$ and depths between 10 and $300 \mathrm{~nm}$, both of which are well within the generally accepted capability of SIMS.

The primary requirement of any measurement technique in process control applications is repeatability. Figure 5 shows excellent repeatability among atom probe depth profiles and bulk concentration measurements from six separate analyses of the same sample, a silicon wafer with a shallow implant of mono-isotopic boron and arsenic. The map (figure 5, left) of the implanted wafer is oriented to provide a view of the data similar to a cross-sectional TEM micrograph. The silicon atoms in the substrate are rendered gray, and the implanted boron and arsenic are rendered blue and purple, respectively. A benign, low-temperature layer was deposited on the top of the wafer to protect the near-surface region of interest during FIB sample preparation. The nickel atoms of this cap are rendered in green. The overlaid depth profiles for boron (figure 5, middle) show good repeatability. "Dose", the critical process parameter, can be determined directly from atom probe data by simply counting the impurity atoms that project onto a square nanometer of the wafer surface. The plots in figure 5 (right) demonstrate the repeatability of dose measurements for both boron and the co-implanted arsenic.

\section{Application Space}

Once confidence is established in the accuracy and precision of a new measurement technique, broader acceptance of the technique depends on its ability to create value. To date, atom-probe microscopy has found valuable applications in bulk metals, metal coatings, thin films and oxides, dopant profiles, advanced (metal and high $k$ ) gate stacks, and thin film conductors (silicides and silicon germanium). Atom probe analysis occupies an application space bordered by SIMS, $\mathrm{X}$-ray photon spectroscopy (XPS) and TEM. It is differentiated from SIMS and XPS primarily by its high spatial resolution, $\sim 0.5 \mathrm{~nm}$ in X and $\mathrm{Y}$ and $\sim 0.1 \mathrm{~nm}$ in $\mathrm{Z}$. While it occupies much of the same high resolution space as TEM, fundamental differences in the imaging and analysis mechanisms yield unique and often complementary capabilities. Combining atom probe and TEM analysis often allows one technique to elucidate details of structure and composition that cannot be resolved by the other.

TEM images are two-dimensional projections of a threedimensional sample. The sample must be thin enough to transmit electrons, typically less than a $100 \mathrm{~nm}$, but thick enough to be mechanically stable. Even at $100 \mathrm{~nm}$, this thin flat sample, commonly called a lamella, is still hundreds of times thicker than the TEM's lateral resolution and fine detail can easily be obscured by overlying
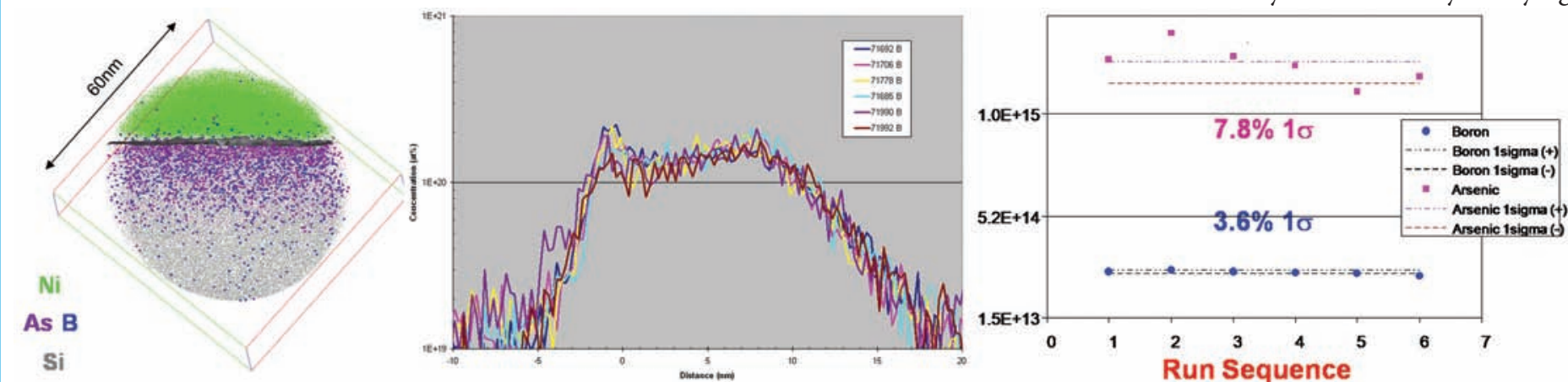

Figure 5 Left - atom map of silicon implanted with boron and arsenic. Middle - overlaid boron depth profiles. Right - Dose measurements for boron and arsenic. 


\section{Atom-Scale Information: Global-Scale Solutions}

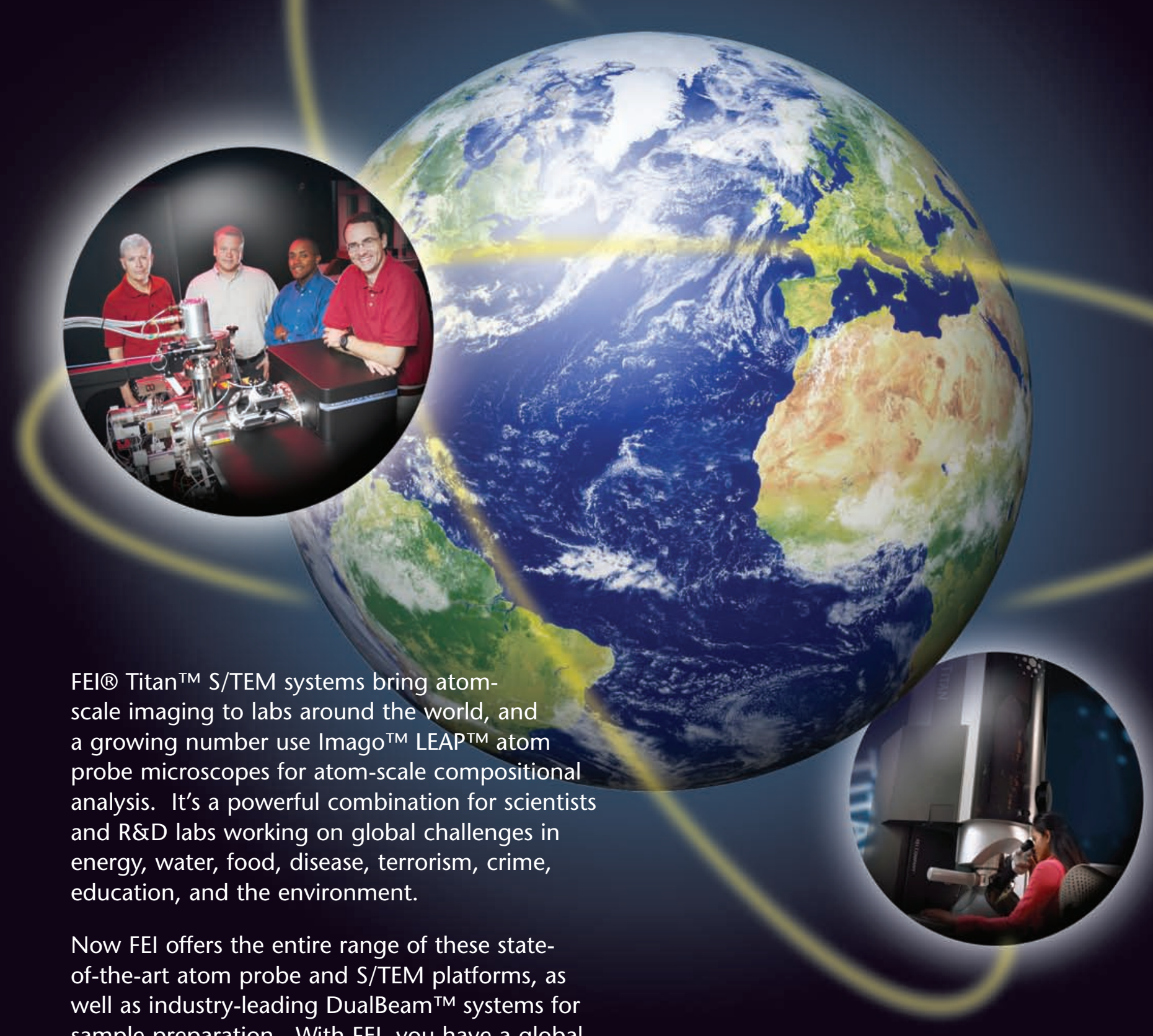
sample preparation. With FEl, you have a global partner to enable you with the most advanced atom-scale technologies for global-scale results.

\section{See more at fei.com}




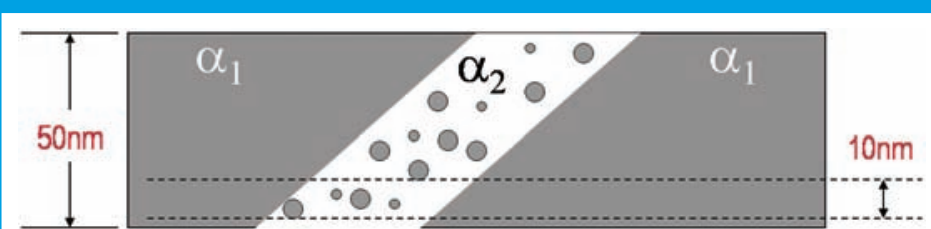

Figure 6 Schematic - A two phase material with precipitates

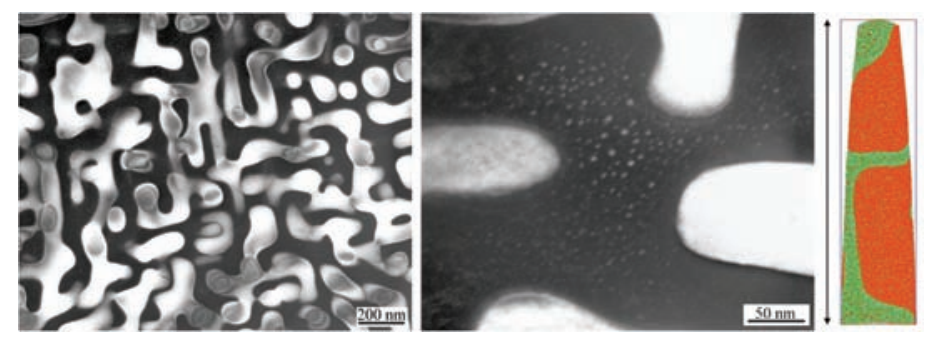

Figure 7 Left - lower magnification DF-TEM [6]. Middle - Higher magnification DF-TEM [6]. Right - 2D projection of $3 D$ atom-probe data.
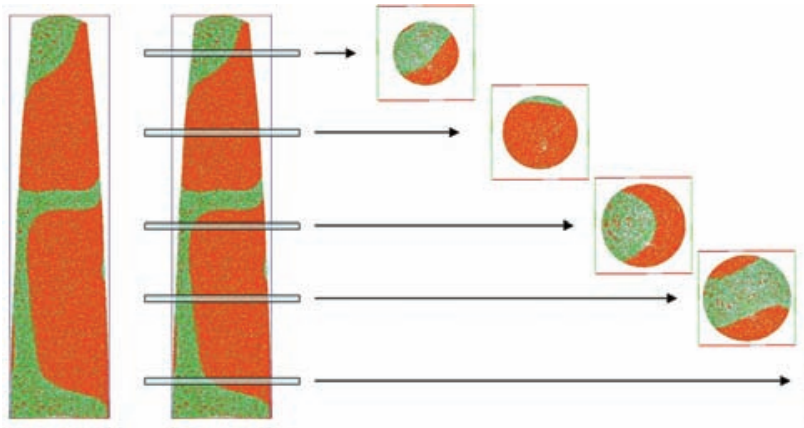

Figure 8 Virtual lamellae

or underlying structure along the direction of the projection. These issues can be addressed to some extent by reducing the thickness of the lamella, and automated FIB techniques have greatly improved the speed and reliability of ultra-thin sample preparation. However, accelerating demand for imaging and analysis of ever smaller structures will be difficult to satisfy with foreseeable advances in the ability to prepare thinner samples.

Atom-probe microscopy examines the sample one ion at a time as each atom (or molecule) ionizes and evaporates from the sample surface. No structure or material ever intervenes between the detected event and the detector. Observations of sub-lamellar structures are independent of sample thickness. The decoded atom probe data, reconstructed into a virtual microstructure of the analyzed volume, can be sliced in any direction, to create a virtual lamella with an arbitrary thickness chosen specifically to isolate and emphasize the structure of interest. While TEM is also capable of three-dimensional modeling using techniques such as tomography and single particle analysis, these methods do not always overcome the limitations created by the thickness of the lamella.
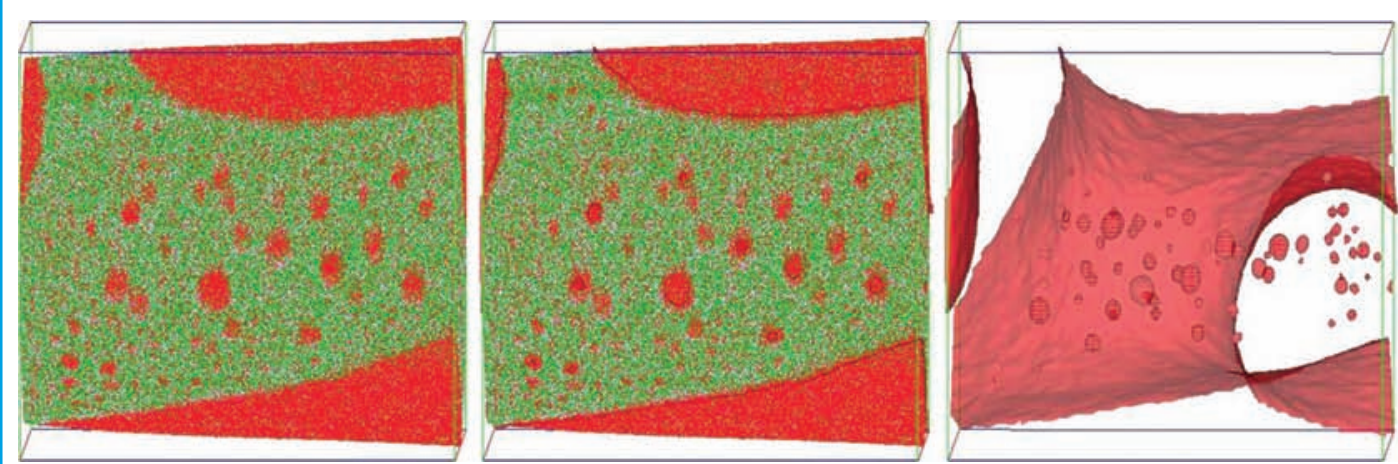

Figure 9 Atom maps Left - display density reduced for transparency. Middle Left - Iso-surfaces at 50\% FE concentration. Middle Right - Iso-surfaces only. Right - precipitates only

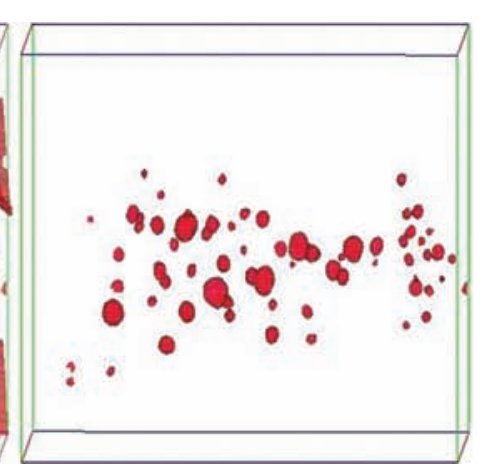

\section{Three Dimensional Atom Probe Analysis - An Example}

Figure 6 presents a schematic view of a situation quite common in the analysis of engineered materials. The sample is a $50 \mathrm{~nm}$ thick volume of a super alloy composed, primarily, of two separate phases. TEM observations have revealed sub-five-nanometer particles finely dispersed throughout one of the phases.

One phase, $\alpha_{1}$, is mostly $\mathrm{Fe}$ with dissolved $\mathrm{Cr}, \mathrm{Ni}$, and $\mathrm{Al}$ and the other phase, $a_{2}$, is NiAl with some dissolved $\mathrm{Cr}$ and Fe. The shape and orientation of the phase regions is arbitrary. Determining the general composition of these major phases is possible using TEM alone. The challenge here is to analyze the small precipitates embedded in the $\alpha_{2}$ phase to determine their composition and morphology. As the dotted lines in figure 7 illustrate, TEM analysis really needs a much thinner $(<10 \mathrm{~nm})$ section to ensure unobscured access to the precipitates. Atom-probe analyzes the full volume of the needle-shaped sample with sub-nm resolution.

Figure 7 shows low and high magnification dark-field TEM (DF-TEM) images of the sample and a two-dimensional projection of an atom-probe data set from a representative sample volume. The TEM dark-field analysis of the precipitated phase is complicated by the small size of the particles. The $\alpha_{1}$ (Fe-rich) phase is bright and the $\alpha_{2}(\mathrm{NiAl})$ phase is dark. The TEM lamella is estimated to be at least $50 \mathrm{~nm}$ thick.

The concept of a virtual lamella (Figure 8) is useful in comparing atom probe and TEM analysis. An atom probe data set is, practically speaking, a virtual microstructure, and selecting a thin, flat volume from the data is analogous to preparing a thin physical lamella from the sample. Of course, defining a virtual lamella $<10 \mathrm{~nm}$ thick is far more practical than fabricating a physical lamella of similar dimensions.

Figure 9 looks at a $90 \mathrm{~nm} \times 90 \mathrm{~nm} \times 100 \mathrm{~nm}$ volume of the sample and demonstrates the power of visual analysis to discover and present important relationships within the rich 3.1 dimensional data set. Left-to-right, the figure shows a progression of maps-twodimensional projections of three-dimensional data-in which the data are manipulated to define and isolate the features of interest, in this case the precipitates. In the first map the appearance of transparency is introduced by displaying only a fraction of the atoms. Fe/Crrich regions are rendered in red tones, while the $\mathrm{Ni} / \mathrm{Al}$-rich regions are rendered in green. In the next map the interfaces are enhanced by the addition of iso-concentration surfaces drawn wherever the Fe concentration is $50 \%$ (inside an iso-concentration surface the composition is $>50 \% \mathrm{Fe}$ ). Next the dots representing the atoms are removed and only the iso-concentration surfaces remain. Finally, the surfaces between the $\alpha 1$ and $\alpha 2$ regions are turned-off leaving only the surfaces that define the precipitates displayed. Clearly the precipitates are iron-rich $(>50 \% \mathrm{Fe})$ and are distributed throughout the NiAl phase. 

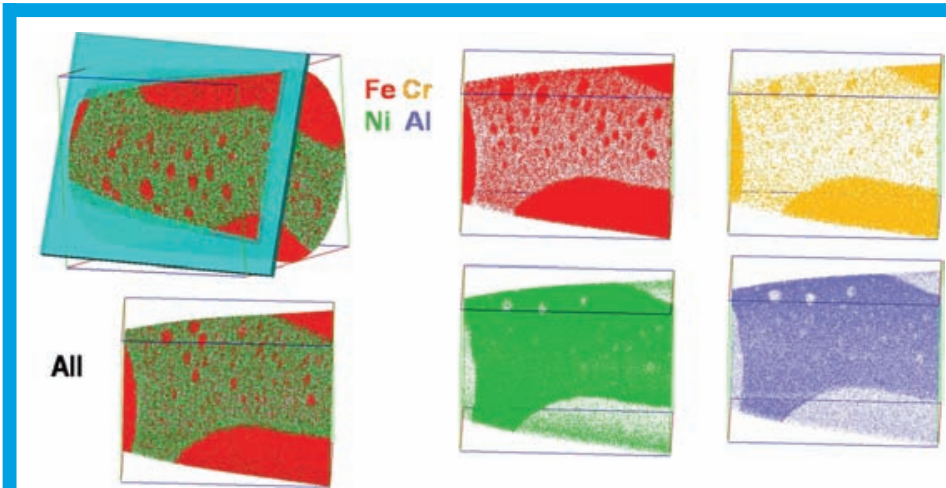
thick

Figure 10 Maps of individual elements in a virtual lamella $5 \mathrm{~nm}$

In figure 10 a $5 \mathrm{~nm}$ virtual lamella (blue parallelepiped with arbitrary orientation) that contains the precipitates has been defined. Projections of the data contained in the lamella for each element reveal that the precipitates are rich in $\mathrm{Cr}$ as well as $\mathrm{Fe}$, and deficient in $\mathrm{Ni}$ and $\mathrm{Al}$.

Analysis of all atoms inside the iso-concentration surfaces (Table 1) determines the average composition of all 71 precipitates in the virtual lamella. The results indicate a composition very similar to that of the $\alpha_{1}$ phase, perhaps with slightly less $\mathrm{Cr}$ and more Ni.

Table 1 Concentration of principal elements in the distinct phases in Figure 10

\begin{tabular}{|l|c|c|c|c|}
\hline at.\% & {$[\mathrm{Fe}]$} & {$[\mathrm{Cr}]$} & {$[\mathrm{Ni}]$} & {$[\mathrm{Al}] \%$} \\
\hline $\begin{array}{l}\mathrm{NiAl}\left(\alpha_{2}\right) \\
\text { phase }\end{array}$ & 11.50 & 1.34 & 44.18 & 43.09 \\
\hline $\begin{array}{l}\text { Fe-rich }\left(\alpha_{1}\right) \\
\text { phase }\end{array}$ & 71.78 & 15.71 & 3.54 & 8.98 \\
\hline $\begin{array}{l}\text { precipitates } \\
\text { (71 total) }\end{array}$ & 76.23 & 9.47 & 8.05 & 6.25 \\
\hline
\end{tabular}

Calculating average composition profiles across the interfaces of all 71 precipitates in the virtual lamella shows increasing Fe and $\mathrm{Cr}$ and decreasing $\mathrm{Ni}$ and $\mathrm{Al}$ concentrations toward the interior (distance $<0 \mathrm{~nm}$ ), Figure 11.

Finally, in Figure 12 the extent of the lamella is reduced to $12 \times 10 \times 5 \mathrm{~nm}$, excluding all but one precipitate. The $50 \% \mathrm{Fe}$ iso-concentration surface delineates the precipitate (left). The $\{100\}$ atomic planes are clearly visible in the $\alpha_{2} \mathrm{NiAl}$ phase (right) as parallel lines. Studying the position of the Fe atoms relative to the $\mathrm{Ni}$ atoms, the Fe-rich precipitate appears to be coherent, sharing the $\{100\}$ lattice spacing with that of the $\alpha_{2}$ matrix. In practice, a $5 \mathrm{~nm}$-thick physical lamella with pristine microstructure is very difficult to achieve. However, the preparation of a $100 \mathrm{~nm}$ diameter atom probe sample is quite straightforward and the extraction of a $5 \mathrm{~nm}$-thick virtual lamella from the data reveals the complementary information that we might not be able to extract from a stand-alone TEM analysis.

\section{Conclusion}

Atom-probe technology has evolved significantly in recent years [8]. It has demonstrated close agreement with accepted imaging and compositional analysis techniques over the targeted range of dimensions and concentrations. A number of applications have been identified where atom-probe analysis is uniquely capable or adds significant value to existing solutions. It occupies an application space that is complementary to TEM, offering similar resolution but

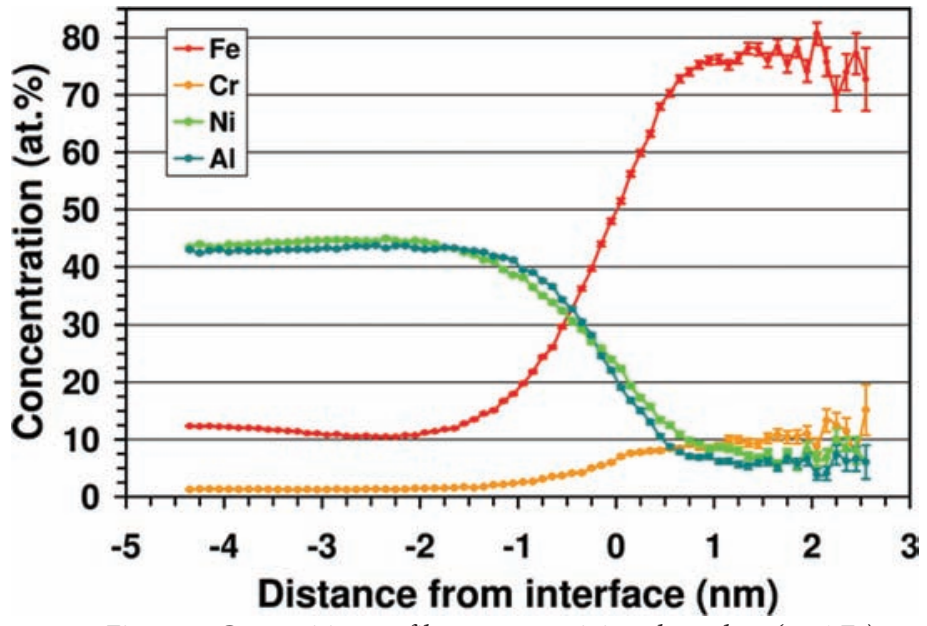

Figure 11 Composition profiles across precipitate boundary $(50 \% \mathrm{Fe})$, left to right is outside to inside
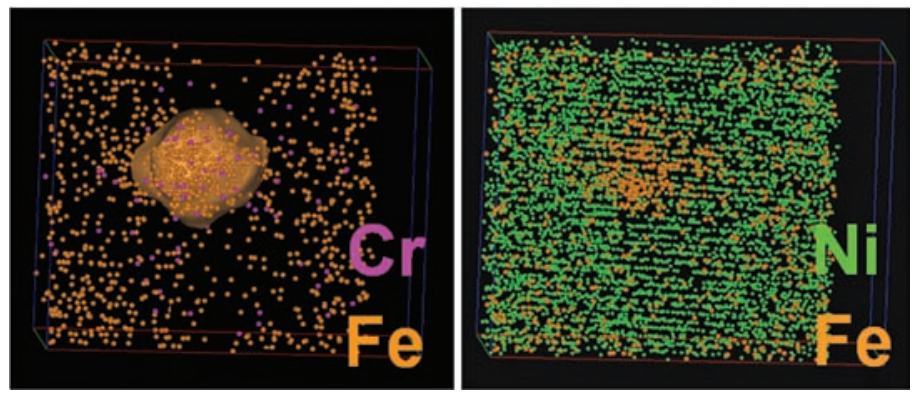

Figure 12 Single precipitate in $5 \mathrm{~nm}$ virtual lamella [7]

well differentiated imaging and analytical capabilities. With easy sample preparation [9] and the elimination of TEM's dependence on sample thickness, it is well positioned to benefit from growing demand to image ever-smaller structures as nanotechnology continues its explosive growth. With credibility established and value adding opportunities identified, atom-probe analysis has entered the analytical mainstream, sine qua non of world class microscopy capability.

\section{References}

1 M.K. Miller, Atom Probe Tomography, Kluwar Academic Publishers, New York, (2000).

2 T.F. Kelly and D.J. Larson, Mat. Char. 44 (2000) 59.

3 T. F. Kelly et al., Micro Microanal. 10(3) (2004) 373.

4 K. Thompson, P. Flaitz, P. Ronsheim, D. J. Larson and T. F. Kelly, Science Sept 7 (2007) 1370.

5 A. Cerezo et al., Materials Today 10(12) (2007).

6 D.J. Larson, T.J. Prosa, S.L.P. Kostrna, M. Ali, T.F. Kelly, C. Stallybrass, A. Schneider, G. Sauthoff and J. Degass, Microscopy and Microanalysis 12(suppl. 2) (2006) $1748 \mathrm{CD}$

7 D. J. Larson and T. F. Kelly, Microscopy and Analysis 78 (2006) 43.

8 T. F. Kelly and M. K. Miller, Rev. Sci. Instrum. 78 (2007) 031101.

9 K. Thompson, D. Lawrence, D. J. Larson, J. D. Olson, T. F. Kelly and B. Gorman, Ultramicroscopy 107(2-3) (2006) 131.

\section{Acknowledgements}

1. Chantal Sudbrack, Northwestern University, and David Reinhard, Peter H Clifton, Ty J. Prosa, Stephan S. A. Gerstl, David J. Larson, Thomas C. Kunicki, Brian P. Geiser, and Edward Oltman, Imago, for Data Analysis

2. Robert M. Ulfig, Dan Lawrence, David Olson, Jesse D. Olson, Joseph H. Bunton, Daniel Chief Lenz, Tye T. Gribb, Timothy Payne, Eric Stennen, Jeffery Shepard, David Sund, Dan Rauls, Duane Kaufman, Terry Walker, and John Watson, Imago, for Data Acquisition

3. C. Stallybrass, G. Sauthoff, A. Schneider, and Y. Degas (Max Planck Institut Düsseldorf) provided the ferritic superalloy material. 